\title{
RÉSONANCE PARAMAGNÉTIQUE ÉLECTRONIQUE ET DICHROISME CIRCULAIRE
}

\author{
par R. SERVANT \\ Laboratoire d'Optique Ultra-Hertzienne de la Faculté des Sciences de Bordeaux
}

\begin{abstract}
Résumé. - A propos du dichroïsme circulaire lié à la résonance paramagnétique électronique, l'auteur, revenant sur la théorie des susceptibilités transverses $\left(\chi_{+}\right)$et $\left(\chi_{-}\right)$en champs tournants, préconise un mode de calcul utilisant les circulaires de Fresnel comme vecteurs de base, dans la représentation des vibrations elliptiques.

Il souligne l'importance relative prise par $\left(\chi_{-}\right)$dans le cas de la résonance très large des sels paramagnétiques en poudres polycristallines.

Abstract. - In connexion with circular dichroïsm bound to electron paramagnetic resonance, the author recalls the theory of rotating field transverse susceptibilities $\left(\chi_{+}\right)$and $\left(\chi_{-}\right)$, then he proposes a way of calculation using Fresnel circulars as basis-vectors in representing elliptic vibrations.

He emphasizes the relative importance taken by $\left(\chi_{-}\right)$in the case of the very wide resonance of polycristalline powder paramagnetic salts.
\end{abstract}

Je voudrais, à propos du dichroïsme circulaire lié à la résonance paramagnétique électronique, dégager la signification des quantités $\left(h_{+}\right),\left(h_{-}\right),\left(m_{+}\right),\left(m_{-}\right)$que l'on est amené à introduire dans le calcul des susceptibilités $\left(\chi_{+}\right)$et $\left(\chi_{-}\right)$en champs tournants $(*)$, car leur utilisation donne parfois lieu à des confusions, et préconiser l'emploi systématique des "vecteurs » de Fresnel, comme vecteurs de base, dans la représentation des vibrations elliptiques.

Je voudrais aussi insister sur l'importance relative prise par $\left(\chi_{-}\right)$dans la résonance large des sels paramagnétiques, en poudres polycristallines.

Sur le calcul des susceptibilités transverses $\left(\chi_{+}\right)$et $\left(\chi_{-}\right)$en champs tournants. - Soit une substance paramagnétique, dont on étudie la résonance paramagnétique électronique. Dans un plan xoy perpendiculaire au champ directeur $H$ (imprimant aux spins un mouvement de précession de vitesse angulaire $\omega_{0}=-\gamma H$ ), la susceptibilité en champ pulsant (de pulsation $\omega$ ) est de la forme :

$$
\chi=\left[\begin{array}{cc}
\chi & -j \xi \\
+j \xi & \chi
\end{array}\right] \text {. }
$$

Les circulaires $\left[\begin{array}{c}1 \\ -j\end{array}\right],\left[\begin{array}{c}1 \\ +j\end{array}\right]$ en sont les directions propres, les valeurs propres correspondantes étant :

$$
\left(\chi_{+}\right)=\chi-\xi \quad\left(\chi_{-}\right)=\chi+\xi .
$$

Le calcul de $\left(\chi_{+}\right)$et $\left(\chi_{-}\right)$s'effectue à partir des 2 premières équations de Bloch, liant l'aimantation transverse $\bar{m}\left(m_{x}, m_{y}\right)$ au champ UHF excitateur $\bar{h}\left(h_{x}, h_{y}\right)$.

Il est commode d'introduire les quantités :

$$
\begin{aligned}
\left(m_{+}\right) & =\left(m_{x}\right)+j\left(m_{y}\right) & \left(m_{-}\right) & =\left(m_{x}\right)-j\left(m_{y}\right) \\
\left(h_{+}\right) & =\left(h_{x}\right)+j\left(h_{y}\right) & \left(h_{-}\right) & =\left(h_{x}\right)-j\left(h_{y}\right)
\end{aligned}
$$

où $\left(m_{x}\right),\left(m_{y}\right),\left(h_{x}\right),\left(h_{y}\right)$ sont les composantes complexes de $\bar{m}$ et de $\bar{h}$, de la forme $a \cdot \exp [j(\omega t-\varphi)](a$ et $\varphi$ pouvant varier d'une composante à l'autre).

(*) Cf par exemple : A. E. Siegman. Microwave Solid-state Masers. New-York, 1964, p. 191.
Comme, d'après l'expression même de $\chi$, on a :

$$
\left(\chi_{+}\right)=\frac{\left(m_{+}\right)}{\left(h_{+}\right)} \quad\left(\chi_{-}\right)=\frac{\left(m_{-}\right)}{\left(h_{-}\right)},
$$

le calcul permet de tirer $\left(\chi_{+}\right)$et $\left(\chi_{-}\right)$.

Interprétation des quantités $\left(h_{+}\right),\left(h_{-}\right),\left(m_{+}\right),\left(m_{-}\right) .-$ Ce qui prècède ne fait pas apparaître la signification physique de ces diverses quantités. Envisageons, au contraire, les 2 circulaires inverses de Fresnel, de rayon $\frac{1}{2}$ :

$$
\left|C_{+}\right\rangle=\frac{1}{2}\left[\begin{array}{c}
1 \\
-j
\end{array}\right] \quad\left|C_{-}\right\rangle=\frac{1}{2}\left[\begin{array}{c}
1 \\
-j
\end{array}\right] .
$$

Un champ excitateur $\bar{h}$ (de pulsation $\omega$ ) pourra toujours, qu'il soit rectiligne, circulaire ou elliptique, être représenté par le vecteur $\mathrm{K}$ et correspondant :

$$
\mid h>=\left[\begin{array}{l}
\left(h_{x}\right) \\
\left(h_{y}\right)
\end{array}\right] \text {. }
$$

On voit immédiatement qu'il pourra s'écrire :

$$
|h\rangle=\left(h_{+}\right)\left|C_{+}>+\left(h_{-}\right)\right| C_{-}>\text {. }
$$

Ainsi, les quantités $\left(h_{+}\right)$et $\left(h_{-}\right)$apparaissent comme les composantes complexes de $\bar{h}$ sur les vecteurs de Fresnel $\mid C_{+}>$et $\mid C_{-}>$.

C'est d'ailleurs ces quantités que nous aurions trouvées (à un facteur près) comme composantes de $\bar{h}$ dans le nouveau système d'axes formé par les directions propres orthogonales $\left[\begin{array}{c}1 \\ -j\end{array}\right],\left[\begin{array}{c}1 \\ +j\end{array}\right]$, qu'il faut adopter pour diagonaliser la matrice $\chi$ et aboutir à :

$$
\left[\begin{array}{l}
\left(m_{+}\right) \\
\left(m_{-}\right)
\end{array}\right]=\left[\begin{array}{cc}
\left(\chi_{+}\right) & 0 \\
0 & \left(\chi_{-}\right)
\end{array}\right]\left[\begin{array}{l}
\left(h_{+}\right) \\
\left(h_{-}\right)
\end{array}\right] .
$$

Remarquons, cependant, que pour conserver une tradition de l'optique, faisant intervenir, pour une vibration rectiligne d'amplitude maximale donnée, les circulaires de Fresnel de rayon moitié, nous prenons ici 
des vecteurs de base $\left|C_{+}\right\rangle$et $\left|C_{-}\right\rangle$qui ne sont pas normés à 1 , au sens hermitique du terme.

Aimantations transverses en tout point de la substance. - Dans le mode de présentation préconisé, l'aimantation transversale s'écrit :

$$
\left|m>=\left(\chi_{+}\right)\left(h_{+}\right)\right| C_{+}>+\left(\chi_{-}\right)\left(h_{-}\right)\left|C_{-}\right\rangle
$$

(qui est bien de la forme $\left(m_{+}\right)\left|C_{+}>+\left(m_{-}\right)\right| C_{-}>$).

Remplaçant $\left(h_{+}\right)$et $\left(h_{-}\right)$par leurs valeurs en fonction de $\left(h_{x}\right)$ et $\left(h_{y}\right)$, et transposant l'égalité sous forme matricielle, on retrouve l'expression de $\chi$ avec :

$$
\chi=\frac{\left(\chi_{-}\right)+\left(\chi_{+}\right)}{2} \quad \xi=\frac{\left(\chi_{-}\right)-\left(\chi_{+}\right)}{2} .
$$

Quant à $\left(\chi_{+}\right)$et $\left(\chi_{-}\right)$, les « équations de Bloch modifiées » permettent, par exemple, d'en trouver la valeur (dans le cas Lorentzien), dans le domaine de linéarité où $M_{z} \simeq M_{0}$ (soit $\gamma^{2} H_{1}^{2} T_{1} T_{2} \ll 1$ ). On trouve pour $\left(\chi_{+}\right)$une expression résonnante (pour $\omega=\omega_{0}$ ) et pour $\left(\chi_{-}\right)$une expression non résonnante dans le domaine des $\omega$ positifs.

Il est important de noter que, dans le calcul, on est amené à écrire des égalités telles que :

$$
\frac{\mathrm{d}\left(m_{ \pm}\right)}{\mathrm{d} t}=j \omega\left(m_{ \pm}\right) \quad(\text { avec le signe }+) .
$$

Il n'en serait pas de même si l'on avait introduit des quantités telles que $\left(\mathcal{H}_{ \pm}\right)=\left(\mathcal{N}_{ \pm}\right) \cdot \mathrm{e}^{ \pm j \omega t}$ représentant des vecteurs tournants.

Dans le mode de calcul adopté, chaque grandeur complexe représente une composante de vecteur, et non un vecteur, ce qui explique qu'elle puisse être toujours en $\exp (j \omega t)$, quel que soit le sens de rotation du vecteur auquel elle se rapporte.

Propagation, à travers la substance, dans le sens du champ $H$, d'une vibration électromagnétique transverse elliptique. - Les susceptibilités transverses $\left(\chi_{+}\right),\left(\chi_{-}\right)$, en champs tournants, étant différentes, il en résulte pour les ondes électromagnétiques à polarisation circulaire, se propageant dans la direction $\mathrm{Oz}$, des constantes de propagation distinctes $\left(\gamma_{ \pm}\right)$.

Examinons, dans ces conditions, comment se modifie une vibration incidente elliptique, lors de sa traversée d'une épaisseur donnée de substance, le long de Oz.

Soit :

$$
|s\rangle=\left(s_{+}\right)\left|C_{+}\right\rangle+\left(s_{-}\right)\left|C_{-}\right\rangle
$$

cette vibration.

Posant :

$$
\begin{aligned}
& \left(s_{+}\right)=\exp \left(-A_{+}\right)=\exp \left[-\left(a_{+}+j b_{+}\right)\right] \\
& \left(s_{-}\right)=\exp \left(-A_{-}\right)=\exp \left[-\left(a_{-}+j b_{-}\right)\right]
\end{aligned}
$$

on voit facilement (du moins pour les ellipticités faibles) que l'inclinaison $R$ du grand axe de l'ellipse et son ellipticité $E$ sont données par :

$$
R=\frac{b_{-}-b_{+}}{2} \quad E=\frac{a_{-}-a_{+}}{2}
$$

ce qui correspond à une rotation complexe :

$$
(\theta)=R-j E=-j \frac{A_{-}-A_{+}}{2} .
$$

Après traversée du milieu dichroïque, sur une longueur $\mathrm{z}$, la vibration $\mid s>$ sera transformée en :

$$
\begin{aligned}
\exp \left[-\left(\gamma_{+}\right) z\right] \cdot\left(s_{+}\right) \mid C_{+} & >+ \\
+ & \exp \left[-\left(\gamma_{-}\right) z\right] \cdot\left(s_{-}\right) \mid C_{-}>.
\end{aligned}
$$

La vibration émergente correspondra aonc à une rotation complexe

$$
-j \frac{\left[A_{-}+\left(\gamma_{-}\right) z\right]-\left[A_{+}+\left(\gamma_{+}\right) z\right]}{2} .
$$

Le supplément de rotation et d'ellipticité, constituant l'effet Faraday complexe lié à la R. P. E. sera donc :

$$
(r)=-j \frac{\left(\gamma_{-}\right)-\left(\gamma_{+}\right)}{2} z \text {. }
$$

Importances relatives de $\chi_{+}^{\prime \prime}$ et $\chi_{-}^{\prime \prime}$. - Il est courant de dire que le dichroïsme circulaire magnétique, associé à la R. P. E., constitue un cas « extrême » de dichroïsme. Il faut entendre par là que les vibrations circulaires de même sens que le courant magnétisant sont absorbées (partiellement) tandis que les vibrations circulaires de sens inverse ne le sont pratiquement pas.

Cependant, il est important, je crois, de souligner que ceci cesse d'être totalement exact dans le cas de signaux R. P. E. très larges, où le produit $\omega T_{2}\left(T_{2}\right.$ étant le temps de relaxation transverse) n'est plus que de quelques unités.

Alors $\chi^{\prime \prime}$, et a fortiori $\chi^{\prime}$ - qui « s'apodise » beaucoup moins vite, ne sont plus totalement négligeables. La figure 1 schématise le mécanisme d'intervention de $\chi_{-}^{\prime \prime}$.
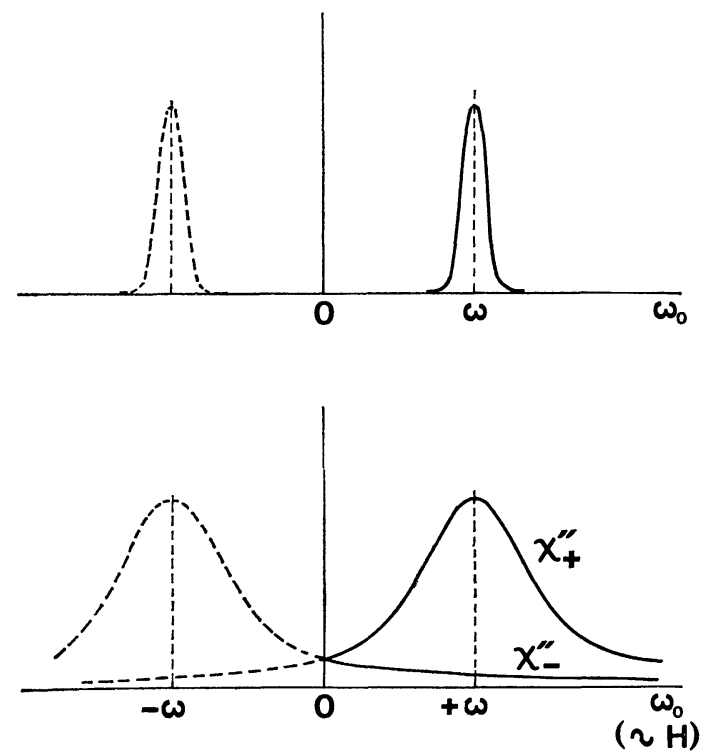

FIG. 1.

Les caractéristiques des phénomènes magnéto-hertziens associés à la R. P. E. prévu par A. Kastler et souvent étudiés depuis, sont liées à l'existence même de cette intervention. 